\title{
Índice de heterogeneidade, coeficiente de variação e tamanho ótimo de parcela em batata
}

\author{
Heterogeneity index, variation coefficient and optimal potato plot size
}

\author{
Sérgio José Ribeiro de Oliveira ${ }^{1}$ Lindolfo Storck $^{2 *}$ \\ Alessandro Dal'Col Lúcio² Sidinei José Lopes² \\ Luiz Fernando Dias Martini ${ }^{3}$
}

\section{RESUMO}

O objetivo deste trabalho foi avaliar a relação entre o coeficiente de variação das parcelas de uma cova e o indice de heterogeneidade com o tamanho ótimo de parcela para experimentos com batata. Foram coletados os dados da produção de tubérculos de batata em doze ensaios. Em cada ensaio, de 12 linhas com 24 covas, foram planejados 18 tipos de parcelas com variações no número de linhas e de comprimentos. Foram ajustadas as funções $C V(x)=A / X^{B} e$ $V U(x)=V 1 / X^{b}$, sendo $X$ o tamanho da parcela em número de unidades básicas, $C V(x)$ o coeficiente de variação entre as parcelas com $X$ unidades básicas e $V U(x)$ a variância por unidade básica entre as parcelas com $X$ unidades básicas. A estimativa do tamanho ótimo de parcela foi procedida por três métodos e os resultados foram correlacionados com as estimativas dos respectivos parâmetros das duas funções. A aplicação da análise de causa e efeito permitiu concluir que o tamanho ótimo de parcela obtido em ensaios em branco, com a cultura da batata, é mais influenciado pelo valor do coeficiente de variação entre as parcelas de uma unidade básica do que pelo índice de heterogeneidade da produtividade.

Palavras-chave: Solanum tuberosum L., índice de he te rogeneidade, planejamento experimental, precisão experimental.

\section{ABSTRACT}

This research was aimed at evaluating the relationship between one plot coefficient variation and heterogeneity index with the optimal potato plot size. Tuber yield data was gotten from 12 potato homogeneity trials. Each trial had 12 rows and 24 hills, from which 18 plot sizes, differing in row and hill numbers, were designed. The functions $C V(x)=A$ / $X^{B}$ and $V U(x)=V 1 / X^{b}$ were adjusted, where $X$ is the plot size in number of basic units $(B U), C V(x)$ is the coefficient of variation between plots with $X B U$, and $V U(x)$ is the $B U$ variation between plots with $X B U$. Optimal plot size estimated by three different methods and the results were correlated with the parameter estimations of the above two functions. Based upon path analysis, the variation coefficient between plots of the basic unit affects more the estimation of optimal plot size than the heterogeneity index in potato uniformity trials.

Key words: Solanum tuberosum L., heterogeneity index, experimental design, experimental precision.

\section{INTRODUÇÃO}

A busca pelo aumento da produtividade no cultivo de batata demanda maior trabalho nas áreas de melhoramento vegetal e técnicas de experimentação. Para se obter experimentos com resultados mais precisos e informações confiáveis, é necessário reduzir o efeito do erro experimental. O erro experimental é causado por vários fatores que conduzem à heterogeneidade do material experimental, à competição intra e interparcelar e à heterogeneidade do solo (STORCK et al., 2000), como também as anormalidades edáficas, agravadas em regiões sob efeitos de clima tropical (RAMALHO et al., 2000).

Uma das formas de se conhecer a heterogeneidade de uma área experimental é implantação e condução de um ensaio de uniformidade (GOMEZ \& GOMEZ, 1984). Isto possibilita observar

\footnotetext{
${ }^{1}$ Departamento de Ciências Exatas e Tecnológicas, Universidade Estadual de Santa Cruz (UESC), 45650-000, Ilhéus, BA, Brasil. ${ }^{2}$ Departamento de Fitotecnia, Universidade Federal de Santa Maria (UFSM), 97105-900, Santa Maria, RS, Brasil. E-mail: lindolfo@smail.ufsm.br.*Autor para correspondência.

${ }^{3}$ Curso de Agronomia, UFSM, Santa Maria, RS, Brasil.
} 
diferenças de fertilidade que poderão ser expressas a partir de diferenças na produtividade das culturas. A existência de distinções entre parcelas vizinhas é atribuída, por alguns autores, à heterogeneidade dos adubos, sementes, mudas, solo, entre outros (STEEL et al., 1997; GOMEZ \& GOMEZ, 1984).

É possível melhorar a precisão experimental buscando-se combinações mais eficientes entre o tamanho e a forma das parcelas, o número de repetições e a diferença mínima significativa entre tratamentos, usando-se o método descrito por HATHEWAY (1961). Nesse método, o pesquisador atende às condições convenientes à pesquisa a partir do prévio conhecimento do tamanho ótimo de parcela e do índice de heterogeneidade da produtividade. Dentre os métodos para estimar o tamanho ótimo de parcelas, destacam-se o método da Máxima Curvatura Modificado (MEIER \& LESSMAN, 1971) da função $C V(x)=A / X^{B}$ e dois métodos descritos por THOMAS (1974) a partir da funções $V U(x)=V_{l} / X^{b} \mathrm{e}$ $C V(x)=V_{1} /\left(M_{1} \sqrt{X^{b}}\right)$, onde $C V(x)$ é o coeficiente de variação entre as parcelas de $\mathrm{X}$ unidades básicas (UB) de tamanho, VU(x) é a variância por UB (SMITH, 1938), $M_{1}$ é a estimativa da média do rendimento entre as parcelas de uma UB, b é o índice de heterogeneidade ou correlação entre as parcelas adjacentes e $V_{1}$ é a variância entre as parcelas de uma UB. Valores de $b$ próximos à unidade indicam alta heterogeneidade da área ou baixa correlação entre parcelas adjacentes. Quando isto ocorre, recomenda-se o uso de parcelas maiores (LIN \& BINNS, 1986).

A persistência das estimativas do índice de heterogeneidade da produtividade de plantas cultivadas em um mesmo local e em diferentes anos é de, aproximadamente, $50 \%$ (LIN et al., 1996). Como conseqüência, a execução de ensaios futuros corretamente planejados quanto ao tamanho de parcela e no número de repetições tem validade na mesma proporção. Trabalhos recentes enfatizam a necessidade de se utilizar mais de um método para determinar o tamanho de parcela (OLIVEIRA, 1994; ZANON, 1996; RESENDE \& SOUZAJÚNIOR, 1997; SILVAet al., 2003; ALVES \& SERAPHIN, 2004). Isso porque, para um mesmo conjunto de dados e uma mesma variável estudada, os métodos poderão mostrar diferentes estimativas de tamanho de parcela, conforme ocorreu em ensaios em branco com batata (OLIVEIRA, 1994). As diferenças nas estimativas do tamanho ótimo de parcelas, obtidos por diferentes métodos, podem resultar das diferentes relações entre as estimativas de parâmetros $\left(\mathrm{A}, \mathrm{B}, \mathrm{V}_{1}, \mathrm{~b}, \mathrm{M}_{1}\right)$ e o tamanho ótimo de parcela para os diferentes ensaios de uniformidade. Neste sentido, alguns estudos mostram as variações e as inter-relações do coeficiente de correlação intraclasse, do índice de heterogeneidade e do coeficiente de variação para a estimação do tamanho de parcela (GOMES, 2002; SILVA, 2003; ALVES \& SERAPHIN, 2004). Assim, a opção por um determinado método poderá influenciar no tamanho da área experimental e no número de repetições necessárias para a discriminação de tratamentos.

Desta forma, o objetivo deste trabalho foi o de avaliar a relação entre o coeficiente de variação das parcelas de uma cova e do índice de heterogeneidade do solo com o tamanho ótimo de parcela para experimentos com batata.

\section{MATERIAL E MÉTODOS}

Doze ensaios de uniformidade de batata da variedade macaca foram conduzidos na estação experimental da Fundação Estadual de Pesquisa Agropecuária (FEPAGRO), em Júlio de Castilhos, Rio Grande do Sul. O solo é o latossolo vermelho escuro distrófico. A área recebeu tratos culturais e fitossanitários uniformes, e a colheita e a pesagem dos tubérculos foram procedidas nos dias 28/11 e 01/12 de 2003.

Cada ensaio foi constituído de 12 linhas, espaçadas em $80 \mathrm{~cm}$, com 24 covas, espaçadas, em média, em $25 \mathrm{~cm}$. Cada cova de batata foi definida como sendo uma unidade básica (UB), ficando identificada pelo número de ordem da linha e da coluna. Planejaramse, para cada um dos 12 ensaios, 18 tipos distintos de parcelas, com diferentes números de linhas (L) e colunas (C), sendo $\mathrm{L} \leq \mathrm{C}$, com os respectivos números de repetições $(\mathrm{N})$, dispostos na tabela 1. Para cada tipo de parcela (tamanho $\mathrm{X}=\mathrm{L}^{*} \mathrm{C}$ ), determinou-se para a característica produtividade de tubérculos: $\mathrm{V}(\mathrm{x})$, estimativa da variância entre as parcelas de X UB de tamanho; $\mathrm{VU}(\mathrm{x})=\mathrm{V}(\mathrm{x}) / \mathrm{X}^{2}$, estimativa da variância por UB entre as parcelas de X UB de tamanho; M(x), estimativa da média das parcelas de X UB de tamanho, em que $M_{1}$ é a média da parcela de uma UB; e CV(x), coeficiente de variação entre as parcelas de X UB de tamanho. Com base nestas determinações, estimou-se o índice de heterogeneidade da produtividade a partir da relação de SMITH (1938), $V U(x)=V_{l} / X^{b}$. Os parâmetros $\mathrm{V}_{1}$ e b foram estimados mediante a transformação logarítmica da função e da ponderação pelos graus de liberdade (STEEL et al., 1997). De forma semelhante, procedeu-se à estimação dos parâmetros A e B da função $C V(x)=A / X^{B}$. Além das estimativas já definidas, também foram determinados os coeficientes de determinação $\left(\mathrm{R}^{2}\right)$ e as estimativas de tamanhos ótimos de parcelas $\left(\mathrm{X}_{0}\right)$ : 
Tabela 1 - Coeficientes de variação e variância por unidade básica entre parcelas de diferentes tamanhos em número de unidades básicas de linhas (L) e de comprimentos (C) e número de repetições (N), em doze ensaios. Santa Maria, 2004.

\begin{tabular}{|c|c|c|c|c|c|c|c|c|c|c|c|c|c|c|}
\hline \multirow[b]{2}{*}{$\mathrm{L}$} & \multirow[b]{2}{*}{$\mathrm{C}$} & \multirow[b]{2}{*}{$\mathrm{N}$} & \multicolumn{12}{|c|}{ Ensaio } \\
\hline & & & 1 & 2 & 3 & 4 & 5 & 6 & 7 & 8 & 9 & 10 & 11 & 12 \\
\hline & & & \multicolumn{12}{|c|}{ Coeficiente de variação } \\
\hline 1 & 1 & 288 & 33,0 & 26,8 & 33,2 & 28,4 & 43,5 & 25,3 & 45,2 & 30,0 & 39,8 & 32,6 & 34,0 & 39,9 \\
\hline 1 & 2 & 144 & 23,5 & 19,8 & 25,1 & 19,7 & 35,0 & 18,6 & 36,9 & 23,3 & 32,4 & 26,3 & 28,7 & 33,1 \\
\hline 1 & 3 & 96 & 20,8 & 16,8 & 22,0 & 17,8 & 32,8 & 17,6 & 32,9 & 22,3 & 29,2 & 24,6 & 27,9 & 30,4 \\
\hline 1 & 4 & 72 & 19,5 & 16,1 & 20,7 & 15,8 & 29,8 & 15,9 & 30,3 & 19,9 & 27,8 & 23,9 & 26,6 & 29,0 \\
\hline 1 & 6 & 48 & 17,8 & 13,8 & 18,9 & 14,4 & 28,5 & 14,2 & 26,9 & 19,3 & 26,5 & 22,5 & 26,2 & 27,7 \\
\hline 1 & 8 & 36 & 17,2 & 13,5 & 17,1 & 12,7 & 27,6 & 13,4 & 26,7 & 17,7 & 26,0 & 21,8 & 25,4 & 26,8 \\
\hline 2 & 2 & 72 & 17,3 & 14,6 & 19,0 & 14,8 & 23,3 & 15,1 & 25,4 & 19,1 & 20,7 & 19,4 & 18,8 & 25,3 \\
\hline 2 & 3 & 48 & 15,2 & 13,1 & 15,9 & 13,5 & 22,4 & 14,3 & 23,0 & 19,1 & 17,8 & 18,9 & 17,8 & 22,5 \\
\hline 2 & 4 & 36 & 14,4 & 12,7 & 14,8 & 12,1 & 19,9 & 13,6 & 21,0 & 17,8 & 16,7 & 18,2 & 16,8 & 22,2 \\
\hline 2 & 6 & 24 & 13,5 & 11,4 & 13,8 & 11,1 & 19,2 & 12,5 & 19,2 & 17,0 & 15,1 & 18,0 & 16,7 & 21,3 \\
\hline 2 & 8 & 18 & 13,7 & 11,0 & 11,9 & 10,2 & 18,8 & 11,5 & 18,2 & 16,2 & 15,0 & 17,7 & 15,9 & 20,8 \\
\hline 3 & 3 & 32 & 13,9 & 11,2 & 13,8 & 12,0 & 20,7 & 9,3 & 18,8 & 15,1 & 9,8 & 17,9 & 10,6 & 17,0 \\
\hline 3 & 4 & 24 & 12,9 & 10,9 & 12,7 & 10,8 & 18,7 & 8,4 & 16,5 & 14,0 & 9,2 & 17,8 & 9,5 & 16,2 \\
\hline 3 & 6 & 16 & 12,8 & 9,9 & 12,4 & 10,1 & 18,0 & 7,7 & 14,5 & 13,2 & 7,9 & 17,2 & 9,0 & 15,4 \\
\hline 3 & 8 & 12 & 12,2 & 10,2 & 10,9 & 9,0 & 17,9 & 7,2 & 12,6 & 12,4 & 6,4 & 17,8 & 7,6 & 14,7 \\
\hline 4 & 4 & 18 & 8,0 & 8,0 & 11,2 & 7,0 & 17,0 & 6,0 & 11,8 & 7,3 & 12,3 & 7,2 & 12,8 & 11,1 \\
\hline 4 & 6 & 12 & 8,0 & 6,7 & 11,3 & 6,2 & 16,2 & 5,0 & 11,9 & 7,1 & 11,1 & 7,3 & 13,3 & 10,2 \\
\hline 4 & 8 & 9 & 7,8 & 7,0 & 9,6 & 4,7 & 16,3 & 2,5 & 9,5 & 5,3 & 10,9 & 7,0 & 12,3 & 9,3 \\
\hline
\end{tabular}

\begin{tabular}{|c|c|c|c|c|c|c|c|c|c|c|c|c|c|c|}
\hline 1 & 1 & 288 & 0,0736 & 0,0604 & 0,0639 & 0,0691 & 0,0854 & 0,0539 & 0,0894 & 0,0770 & 0,0944 & 0,0969 & 0,0930 & 0,1341 \\
\hline 1 & 2 & 144 & 0,0371 & 0,0329 & 0,0364 & 0,0330 & 0,0552 & 0,0292 & 0,0598 & 0,0462 & 0,0626 & 0,0631 & 0,0665 & 0,0919 \\
\hline 1 & 3 & 96 & 0,0291 & 0,0237 & 0,0281 & 0,0270 & 0,0484 & 0,0259 & 0,0473 & 0,0426 & 0,0509 & 0,0550 & 0,0626 & 0,0775 \\
\hline 1 & 4 & 72 & 0,0255 & 0,0219 & 0,0248 & 0,0213 & 0,0400 & 0,0212 & 0,0403 & 0,0337 & 0,0459 & 0,0522 & 0,0568 & 0,0706 \\
\hline 1 & 6 & 48 & 0,0214 & 0,0159 & 0,0206 & 0,0178 & 0,0368 & 0,0169 & 0,0318 & 0,0317 & 0,0418 & 0,0461 & 0,0555 & 0,0644 \\
\hline 1 & 8 & 36 & 0,0199 & 0,0154 & 0,0169 & 0,0137 & 0,0343 & 0,0151 & 0,0311 & 0,0266 & 0,0401 & 0,0434 & 0,0518 & 0,0605 \\
\hline 2 & 2 & 72 & 0,0201 & 0,0178 & 0,0208 & 0,0186 & 0,0246 & 0,0193 & 0,0283 & 0,0312 & 0,0254 & 0,0344 & 0,0286 & 0,0537 \\
\hline 2 & 3 & 48 & 0,0156 & 0,0143 & 0,0145 & 0,0155 & 0,0226 & 0,0172 & 0,0232 & 0,0311 & 0,0189 & 0,0325 & 0,0256 & 0,0427 \\
\hline 2 & 4 & 36 & 0,0139 & 0,0135 & 0,0127 & 0,0126 & 0,0179 & 0,0155 & 0,0193 & 0,0270 & 0,0166 & 0,0300 & 0,0227 & 0,0413 \\
\hline 2 & 6 & 24 & 0,0123 & 0,0108 & 0,0111 & 0,0105 & 0,0167 & 0,0131 & 0,0162 & 0,0247 & 0,0135 & 0,0294 & 0,0225 & 0,0382 \\
\hline 2 & 8 & 18 & 0,0125 & 0,0102 & 0,0082 & 0,0088 & 0,0159 & 0,0112 & 0,0145 & 0,0225 & 0,0133 & 0,0284 & 0,0205 & 0,0363 \\
\hline 3 & 3 & 32 & 0,0129 & 0,0106 & 0,0109 & 0,0123 & 0,0193 & 0,0073 & 0,0154 & 0,0194 & 0,0057 & 0,0293 & 0,0091 & 0,0243 \\
\hline 3 & 4 & 24 & 0,0112 & 0,0099 & 0,0094 & 0,0099 & 0,0157 & 0,0059 & 0,0119 & 0,0167 & 0,0050 & 0,0289 & 0,0073 & 0,0221 \\
\hline 3 & 6 & 16 & 0,0111 & 0,0082 & 0,0089 & 0,0088 & 0,0146 & 0,0049 & 0,0092 & 0,0148 & 0,0037 & 0,0267 & 0,0065 & 0,0199 \\
\hline 3 & 8 & 12 & 0,0099 & 0,0087 & 0,0068 & 0,0069 & 0,0144 & 0,0043 & 0,0069 & 0,0130 & 0,0025 & 0,0289 & 0,0046 & 0,0181 \\
\hline 4 & 4 & 18 & 0,0043 & 0,0053 & 0,0072 & 0,0042 & 0,0130 & 0,0030 & 0,0061 & 0,0045 & 0,0090 & 0,0047 & 0,0132 & 0,0103 \\
\hline 4 & 6 & 12 & 0,0043 & 0,0037 & 0,0074 & 0,0033 & 0,0118 & 0,0020 & 0,0062 & 0,0043 & 0,0073 & 0,0048 & 0,0142 & 0,0087 \\
\hline 4 & 8 & 9 & 0,0040 & 0,0041 & 0,0054 & 0,0019 & 0,0121 & 0,0005 & 0,0039 & 0,0023 & 0,0071 & 0,0044 & 0,0122 & 0,0072 \\
\hline
\end{tabular}

$$
X_{0}=\exp \left\{\left(\frac{1}{2 B+2}\right) \ln \left[\frac{A^{2} B^{2}(2 B+1)}{B+2}\right]\right\}
$$

, método MO (MEIER \& LESSMAN, 1971);

$$
X_{0}=\exp \left\{\left(\frac{1}{2 b+2}\right) \ln \left[\frac{b V_{1}^{2}(2 b+1)}{b+2}\right]\right\}
$$

método MV (THOMAS, 1974); e
$X_{0}=\exp \left\{\left(\frac{1}{b+2}\right) \ln \left[\frac{b^{2} V_{1}(b+1)}{2(b+4) M_{1}^{2}}\right]\right\}$

método MC (THOMAS, 1974).

Estimaram-se as correlações lineares entre as estimativas dos parâmetros $\left(\mathrm{A}, \mathrm{B}, \mathrm{V}_{1}, \mathrm{~b}, \mathrm{M}_{1}\right)$ e o tamanho ótimo de parcela $\left(\mathrm{X}_{0}\right)$ obtido pelos três métodos (MO, MV e MC). Com o objetivo de verificar 
quais as estimativas de parâmetros interferem direta ou indiretamente no valor de $\mathrm{X}$, procedeu-se à análise de causa e efeito (trilha) (VENCOVSKY \& BARRIGA, 1992; CRUZ \& REGAZZI, 1994). Assim, para X estimado pelo método MO, a análise de trilha foi realizada entre $\mathrm{X}_{0}$ (variável principal) e as estimativas de A e B (variáveis explicativas). Para o método MV, a análise de trilha foi realizada entre $\mathrm{X}_{0}$ com b e $\mathrm{V}_{1}$; e para o método MC, entre $\mathrm{X}_{0}$ com b $\mathrm{V}_{1}$ e $\mathrm{M}_{1}$. Para os cálculos estatísticos, foram utilizados os seguintes pacotes estatísticos: o software científico - NTIA, desenvolvido pelo Centro Tecnológico para Informática (EMBRAPA, 1997), o programa GENES (CRUZ, 2001), o programa SAEG (UFV, 2005) e um programa em linguagem Fortran (ABOU-EL-FITTOUH et al., 1974), com algumas modificações, específico para calcular a média e as variâncias de parcelas de diferentes tamanhos.

\section{RESULTADOS E DISCUSSÃO}

Os coeficientes de variação e as variâncias por unidade básica (Tabela 1) decrescem com o aumento do tamanho das parcelas, para cada um dos doze ensaios de 288 unidades básicas, de acordo com a função ou lei empírica de SMITH (1938). Além disso, observa-se uma variação de $25,3 \%$ a $45,2 \%$ para o $\mathrm{CV}(\mathrm{x}=1)$ e entre $2,5 \%$ e $16,3 \%$ para $\mathrm{CV}(\mathrm{x}=4 * 8=32)$ nos doze ensaios. Assim, existem variações nos valores máximos e mínimos e na forma de redução do CV com o aumento do tamanho das parcelas. Desta forma, o conjunto dos doze ensaios pode ser adequado para o estudo proposto.
A estimativa da origem $\left(\mathrm{V}_{1}\right)$ da função $\mathrm{VU}(\mathrm{X})=\mathrm{V}_{1} / \mathrm{X}^{\mathrm{b}}$ tem maior variabilidade (maior $\mathrm{CV}$ ) do que a estimativa da origem (A) da função $C V(x)=A / X^{B}$, sendo que as estimativas da forma curva (B e b) das mesmas funções tem variabilidade semelhante (Tabela 2). A produtividade de batata variou de 0,6621 a $0,9542 \mathrm{~kg} \mathrm{cova}^{-1}$, com média igual a $0,8452 \mathrm{~kg} \mathrm{cova}^{-1} \mathrm{e}$ coeficiente de variação entre as médias dos ensaios igual a $12,33 \%$. Assim, considerando os CV entre as parcelas de uma UB nos doze ensaios (Tabela 1) com média de $44,75 \%$, a variabilidade da produção de batata está mais dentro dos ensaios do que entre eles. A menor variabilidade entre ensaios mostra a persistência ou a validade do uso dos resultados obtidos para o planejamento de experimentos futuros, o que já foi constatado por LIN et al. (1996).

A média do índice de heterogeneidade nos 12 ensaios $(b=0,9402)$ pode ser considerada alta, confirmando a existência de heterogeneidade ou baixa correlação entre as parcelas adjacentes (GOMEZ \& GOMEZ, 1984; LIN \& BINNS, 1986), e também devido à heterogeneidade das batatas sementes, do adubo, entre outros (STEEL et al., 1997). Este fato leva a inferir, conforme recomendação de estudos (LIN \& BINNS, 1986; ROSSETTI, 2002), que parcelas adequadas devem ter tamanho maior.

As estimativas do tamanho ótimo de parcela $\left(\mathrm{X}_{0}\right)$ foram consideradas viáveis para implantação na prática apenas no método de Máxima Curvatura Modificado (MO), de MEIER \& LESSMAN (1971). Os métodos da Máxima Curvatura de VU(x) (MV) e o de $\mathrm{CV}(\mathrm{x})$ (MC) subestimaram os valores de $\mathrm{X}_{0}$, apresentando valores inferiores a uma UB em todos os 12 ensaios (Tabela 2). Além dos métodos MV e MC

Tabela 2 - Estimativas dos parâmetros das funções $\mathrm{CV}(\mathrm{x})=\mathrm{A} / \mathrm{X}^{\mathrm{B}}$ e $\mathrm{VU}(\mathrm{x})=\mathrm{V}_{1} / \mathrm{X}^{\mathrm{b}}$, coeficientes de determinação $\left(\mathrm{R}^{2}\right)$, média de rendimento de tubérculos de batata $(\mathrm{kg})$ nas parcelas de uma unidade básica $\left(\mathrm{M}_{1}\right)$, usando uma cova por unidade básica, em doze ensaios, média e coeficiente de variação (CV), tamanho ótimo de parcela obtidos pelos métodos de Máxima Curvatura Modificada (MO), Máxima Curvatura da função VU (MV) e da função CV (MC). Santa Maria, 2004.

\begin{tabular}{|c|c|c|c|c|c|c|c|c|c|c|}
\hline \multirow[b]{2}{*}{ Ensaio } & \multicolumn{3}{|c|}{$\mathrm{CV}(\mathrm{x})=\mathrm{A} / \mathrm{X}^{\mathrm{B}}$} & \multicolumn{3}{|c|}{$\mathrm{VU}(\mathrm{x})=\mathrm{V}_{1} / \mathrm{X}^{\mathrm{b}}$} & \multirow[b]{2}{*}{$\mathrm{M}_{1}$} & \multirow[b]{2}{*}{$\mathrm{MO}$} & \multirow[b]{2}{*}{ MV } & \multirow[b]{2}{*}{$\mathrm{MC}$} \\
\hline & A & B & $\mathrm{R}^{2}$ & $\mathrm{~V}_{1}$ & $\mathrm{~b}$ & $\mathrm{R}^{2}$ & & & & \\
\hline 1 & 34,64 & 0,4057 & 0,705 & 0,0809 & 0,8118 & 0,705 & 0,8209 & 5,9245 & 0,2312 & 0,2240 \\
\hline 2 & 27,13 & 0,3767 & 0,780 & 0,0619 & 0,7537 & 0,780 & 0,9169 & 4,8443 & 0,1838 & 0,1709 \\
\hline 3 & 31,01 & 0,3344 & 0,942 & 0,0557 & 0,6686 & 0,942 & 0,7611 & 5,0889 & 0,1509 & 0,1613 \\
\hline 4 & 36,70 & 0,5274 & 0,783 & 0,1154 & 1,0520 & 0,783 & 0,9250 & 6,4997 & 0,3549 & 0,3181 \\
\hline 5 & 38,67 & 0,2217 & 0,819 & 0,0676 & 0,5235 & 0,819 & 0,6723 & 5,3547 & 0,1288 & 0,1392 \\
\hline 6 & 58,81 & 0,7713 & 0,714 & 0,2913 & 1,5422 & 0,714 & 0,9181 & 8,4088 & 0,6894 & 0,6243 \\
\hline 7 & 62,11 & 0,5188 & 0,892 & 0,1692 & 1,0377 & 0,892 & 0,6621 & 9,1765 & 0,4232 & 0,4426 \\
\hline 8 & 55,31 & 0,5872 & 0,643 & 0,2610 & 1,1740 & 0,643 & 0,9240 & 8,4835 & 0,5663 & 0,4659 \\
\hline 9 & 43,16 & 0,4704 & 0,540 & 0,1110 & 0,9408 & 0,540 & 0,7718 & 7,1384 & 0,3155 & 0,3115 \\
\hline 10 & 52,97 & 0,5105 & 0,485 & 0,2554 & 1,0208 & 0,485 & 0,9542 & 8,2611 & 0,5124 & 0,3914 \\
\hline 11 & 35,77 & 0,3699 & 0,447 & 0,1032 & 0,7396 & 0,447 & 0,8982 & 5,8858 & 0,2415 & 0,2040 \\
\hline 12 & 60,80 & 0,5087 & 0,752 & 0,3109 & 1,0174 & 0,752 & 0,9172 & 9,0464 & 0,5636 & 0,4274 \\
\hline Média & 44,75 & 0,4702 & 0,708 & 0,1570 & 0,9402 & 0,708 & 0,8452 & 7,0094 & 0,3635 & 0,3234 \\
\hline $\mathrm{CV}(\%)$ & 27,96 & 28,53 & 21,85 & 61,41 & 28,51 & 21,85 & 12,33 & 22,91 & 51,17 & 46,46 \\
\hline
\end{tabular}


subestimarem $\mathrm{X}_{0}$, não se pode usar parcelas menores do que uma cova; seus resultados são mais heterogêneos, pois têm CV maiores. Observando as expressões algébricas para a estimativa de $\mathrm{X}_{0}$ pelos métodos MV e MC, observa-se que, quando o valor de $\mathrm{V}$ for menor do que a unidade, o valor de $\mathrm{X}_{0}$ também vali ser menor do que a unidade. Nestes casos, bastaria usar os valores em gramas no lugar de quilogramas (usada neste estudo), para resultar em variâncias $\left(\mathrm{V}_{1}\right)$ maiores do que a unidade e $\mathrm{X}_{0}$ maiores do que a unidade. Assim, constata-se uma falha na metodologia de THOMAS (1974), fazendo com que os resultados dependam da escala da unidade de medida usada para a variável. Por outro lado, o método MO (função de A e B) resultou uma média de tamanho ótimo de parcela de sete covas, com variações entre cinco e nove covas, não dependendo da escala de medida porque a estimativa de $\mathrm{A}$ é um percentual e $\mathrm{B}=\mathrm{b} / 2$ é a metade do valor do índice de heterogeneidade.

As estimativas das correlações lineares entre as estimativas de $\mathrm{X}_{0}$ e as estimativas dos parâmetros que estão presentes nas respectivas expressões algébricas que estimam $\mathrm{X}_{0}$ foram positivas e significativas (Tabela 3). Também mostram que a estimativa da média de parcelas de uma UB $\left(M_{1}\right)$ não apresentou associação significativa com nenhuma das demais estimativas, principalmente para a estimativa de A, cujo valor do coeficiente de correlação foi desprezível. As demais estimativas apresentaram associações significativas entre si, inclusive entre os tamanhos ótimos de parcela, para os métodos MO, MV e MC. Destaca-se, entretanto, que as estimativas mais correlacionadas são $\mathrm{b}$ e $\mathrm{B}$, pois b é semelhante a $2 \mathrm{~B}$ (THOMAS, 1974). Pode-se dizer também que, elevando-se os valores das estimativas de A e B, aumenta o valor de $\mathrm{X}_{0}$ para o método $\mathrm{MO}$, assim como havendo incremento nos valores de $\mathrm{V}_{1}$ e $\mathrm{b}$, aumenta o valor de $\mathrm{X}_{0}$ para o método $\mathrm{MV}$, o mesmo ocorrendo entre $\mathrm{V}_{1}$ e b em relação a $\mathrm{X}_{0}$ para o método MC. Estas correlações são coerentes, pois a maior variabilidade entre as UB (maiores valores $\mathrm{A}$ e $\mathrm{V}_{1}$ ) e a forma das funções (b próximo da unidade) sugere a necessidade de se usar parcelas maiores (LIN \& BINNS, 1986; ROSSETTI, 2002).

É possível considerar dois casos de relação entre o índice de heterogeneidade do solo e o grau de dependência de $\mathrm{V}_{1}$ e $\mathrm{X}$, a partir dos valores teóricos extremos de b. Em primeiro lugar, quando o valor de bé igual a zero, a $V_{1}$ entre UB não depende de $\mathrm{X}$; em segundo lugar, quando b está próximo da unidade, $\mathrm{a}_{1}$ dependerá do número de UB por parcela (LIN \& BINNS, 1986). As condições deste experimento, em que os valores de b e B estão próximos aos máximos teóricos (Tabela 2), confirmam essa dependência das estimativas dos parâmetros $\mathrm{A}$ e $\mathrm{V}_{1}$ do número de UB por parcela. Estas considerações também são esperadas para a função $C V(x)=A / X^{B}$; contudo, devido à relação entre as funções $\mathrm{CV}(\mathrm{x})$ e VU(x), deve-se considerar o máximo de $B$ igual a 0,5 .

No caso da aplicação do método da Máxima Curvatura Modificado (MO) para estimar o tamanho ótimo de parcela $\left(\mathrm{X}_{0}\right)$, a estimativa da correlação de $\mathrm{X}_{0}$ com as estimativas de A e B explica a associação existente entre $\mathrm{A}$ e $\mathrm{X}_{0}$, pois o efeito direto (Tabela 4) assemelha-se em magnitude e sinal $(0,88)$ à correlação $(0,9744)$. Já a associação entre a estimativa de B e X 0 é explicada pelo efeito indireto da estimativa de A, o qual dá maior importância para a estimativa de A, que é o coeficiente de variação entre as parcelas de uma UB. Assim, fica comprovado que a variação entre as estimativas de Aé o principal responsável pela variação do valor do tamanho ótimo de parcela e que o valor do índice de heterogeneidade (correlação entre as parcelas adjacentes) tem menor influência. Nestas condições, pressupõe-se que, se o pesquisador aplicar uma

Tabela 3 - Estimativas dos coeficientes de correlação linear entre as variáveis rendimento de tubérculos de batata (média de uma unidade básica $=\mathrm{M}_{1}$ ), estimativas dos parâmetros das funções $\mathrm{CV}(\mathrm{x})=\mathrm{A} / \mathrm{X}^{\mathrm{B}}$ e $\mathrm{VU}(\mathrm{x})=\mathrm{V}_{1} / \mathrm{X}^{\mathrm{b}}$ e estimativas do tamanho ótimo de parcela, usando os métodos de Máxima Curvatura Modificada (MO), Máxima Curvatura em função da VU (MV) e CV (MC) para 12 ensaios. Santa Maria, 2004.

\begin{tabular}{lcllllll}
\hline & $\mathrm{M}_{1}$ & $\mathrm{~A}$ & $\mathrm{~B}$ & $\mathrm{~V}_{1}$ & $\mathrm{~b}$ & $\mathrm{MO}$ & $\mathrm{MV}$ \\
\hline $\mathrm{A}$ & 0,0639 & - & - & - & - & - & - \\
$\mathrm{B}$ & 0,4838 & $0,6933^{*}$ & - & - & - & - & - \\
$\mathrm{V}_{1}$ & 0,4866 & $0,8856^{*}$ & $0,7899^{*}$ & - & - & - & - \\
B & 0,4608 & $0,7121^{*}$ & $0,9999^{*}$ & $0,7898^{*}$ & - & - & - \\
MO & 0,1702 & $0,9744^{*}$ & $0,7549^{*}$ & $0,8874^{*}$ & $0,7557^{*}$ & - & - \\
MV & 0,4898 & $0,8706^{*}$ & $0,9158^{*}$ & $0,9624^{*}$ & $0,9232^{*}$ & $0,8955^{*}$ & - \\
MC & 0,3324 & $0,8802^{*}$ & $0,9579^{*}$ & $0,8893^{*}$ & $0,9589^{*}$ & $0,8949^{*}$ & $0,9727^{*}$ \\
\hline
\end{tabular}

*significativo pelo teste t em nível de $5 \%$ de probabilidade de erro. 
Tabela 4 - Efeitos diretos e indiretos das estimativas dos parâmetros das funções $\mathrm{CV}(\mathrm{x})=\mathrm{A} / \mathrm{X}^{\mathrm{B}}$ e $\mathrm{VU}(\mathrm{x})=$ $\mathrm{V}_{1} / \mathrm{X}^{\mathrm{b}}$ e da média das parcelas com uma unidade básica $\left(\mathrm{M}_{1}\right)$ sobre as estimativas do tamanho ótimo de parcela pelos métodos de Máxima Curvatura Modificada (MO), Máxima Curvatura em função da VU (MV) e de CV (MC) para 12 ensaios. Santa Maria, 2004.

\begin{tabular}{llllll}
\hline Efeito & $\mathrm{A}$ & $\mathrm{B}$ & $\mathrm{V}_{1}$ & $\mathrm{~b}$ & $\mathrm{M}_{1}$ \\
\hline Direto sobre MO & 0,88 & 0,14 & - & - & - \\
Indireto via & 0,61 & 0,10 & - & - & - \\
& - & - & - & - & - \\
Direto sobre MV & - & - & 0,62 & 0,43 & - \\
Indireto via & - & - & 0,49 & 0,34 & - \\
& - & - & - & - & - \\
Direto sobre MC & - & - & 0,42 & 0,72 & $-0,20$ \\
Indireto via & - & - & - & 0,57 & $-0,10$ \\
Indireto via & - & - & 0,33 & - & $-0,09$ \\
Indireto via & - & - & 0,20 & 0,33 & - \\
\hline
\end{tabular}

adubação mais forte na cultura da batata, aumentará a média e, com isto, o coeficiente de variação entre as parcelas de uma UB ficará reduzido e, por decorrência, a estimativa do tamanho ótimo de parcela fica reduzida.

Para o método MV, a estimativa da correlação entre $\mathrm{X}_{0} \mathrm{e} \mathrm{V}_{1}$, assim como entre $\mathrm{X}_{0} \mathrm{e} \mathrm{b}$, apresentam o mesmo sinal (Tabela 3 ), e as magnitudes são semelhantes, havendo equilíbrio dos efeitos diretos e indiretos entre $\mathrm{V}_{1}$ e b em relaç̧ão a $\mathrm{X}_{0}$ (Tabela 4).

No caso do método MC, $\mathrm{b}$ influencia direta e indiretamente o valor de $\mathrm{X}$ (Tabela 4). $\mathrm{V}_{1}$ influencia $\mathrm{X}_{0}$ indiretamente, principalmente via $\mathrm{b}$. $\mathrm{M}_{1}$ influencia indiretamente via $\mathrm{b}$; no entanto, embora a correlação entre $M_{1}$ e $X_{0}$ seja positiva, sua influência apresenta sinal contrário, tanto direta quanto indiretamente, via b e via $\mathrm{V}_{1}$.

Principalmente em relação ao método da Máxima Curvatura Modificado, usado em diversos estudos (MEIER \& LESSMAN, 1971; OLIVEIRA, 1994; SILVAet al., 2003; ZANON, 1996; ALVES \& SERAPHIN, 2004), fica comprovado que se deve dar mais importância para a caracterização da unidade básica (tamanho da UB, manejo, adubação, entre outros) do que simplesmente ao valor do índice de heterogeneidade na área experimental. Assim, a persistência dos resultados obtidos no planejamento dos experimentos futuros depende de serem mantidas as mesmas condições técnicas de cultivo.

\section{CONCLUSÃO}

O tamanho ótimo de parcela obtido em ensaios em branco, com a cultura da batata, é mais influenciado pelo valor do coeficiente de variação entre as parcelas de uma unidade básica do que pelo índice de heterogeneidade da produtividade.

\section{AGRADECIMENTOS}

Ao Programa Institucional de Bolsas de Iniciação Científica/Conselho Nacional de Desenvolvimento Científico e Tecnológico (PIBIC/CNPq), pela concessão de bolsa ao pesquisador Martini e à Fundação de Amparo à Pesquisa do Estado do Rio Grande do Sul (FAPERGS), pelo auxílio financeiro

\section{REFERÊNCIAS}

ABOU-EL-FITTOUH, H.A. et al. A program for studying the optimum plot size in field experiments on the computer. Agricultural Research Review, v.52, p.85-90, 1974.

ALVES, S.M.F.; SERAPHIN, J.C. Coeficiente de heterogeneidade do solo e tamanho de parcela. Pesquisa Agropecuária Brasileira, v.39, p.105-111, 2004.

CRUZ, C.D. Programa GENES - versão windows. Aplicativo computacional em genética e estatística. Viçosa: UFV, 2001. 648p.

CRUZ, C.D.; REGAZZI, A.J. Modelos biométricos aplicados ao melhoramento genético. Viçosa: UFV, 1994. 390p.

EMBRAPA. Ambiente de software NTIA, versão 4.2.2: manual do usuário - ferramental estatístico. Campinas: Centro Nacional de Pesquisa Tecnológica em Informática para a Agricultura, 1997. 258p.

GOMES, F.P. O problema do tamanho ótimo de parcelas experimentais: relação entre o coeficiente b de Smith e o coeficiente de correlação entraclasse $(\rho)$. Revista de Agricultura, v.77, p.385-389, 2002.

GOMEZ, K.A.; GOMEZ, A.A. Statistical procedures for agricultural research. 2.ed. New York: John Wiley, 1984. 680 p.

HATHEWAY, W.H. Convenient plot size. Agronomy Journal, v.53, p.279-280, 1961

LIN, C.S. et al. Persistence of a field heterogeneity index. Canadian Journal of Plant Science, v.76, p.245-250, 1996.

LIN, C.S.; BINNS, M.R. Relative efficiency of two randomized block designs having different plot size and numbers of replications and of plots per block. Agronomy Journal, v.78, p.531-534, 1986.

MEIER, V.D.; LESSMAN, K.J. Estimation of optimum field plot shape and size testing yield in Crambe abyssinica hordnt. Crop Science, v.11, p.648-650, 1971.

OLIVEIRA, P.H. Tamanho e forma ótimos da parcela para avaliação do rendimento em experimentos com batata. 1994. 83f. Dissertação (Mestrado em Agronomia) Universidade Federal de Santa Maria, Santa Maria. 
RAMALHO, M.A.P. et al. A experimentação em genética e melhoramento de plantas. Lavras: EDUFLA, 2000. 326p.

RESENDE, M.D.V.; SOUZA JÚNIOR, C.L. Número de repetições e tamanho da parcela para seleção de progênies de milho em solos sob cerrado e fértil. Pesquisa Agropecuária Brasileira, v.32, p.781-788, 1997.

ROSSETTI, A.G. Influência da área da parcela e do número de repetições na precisão de experimentos com arbóreas. Pesquisa Agropecuária Brasileira, v.37, p.433-438, 2002.

SILVA, R.L. et al. Determinação do tamanho ótimo da parcela experimental pelos métodos da máxima curvatura modificado, do coeficiente de correlação intraclasse e da análise visual em testes clonais de eucalipto. Revista Árvore, v.27, p.669-676, 2003.

SMITH, H.F. An empirical law describing heterogeneity in the yields of agricultural crops. Journal Agricultural Science, n.28, p.1-23, 1938.
STEEL, R.G.D. et al. Principles and procedures of statistics: a biometrical approach. 3.ed. New York: McGraw Hill Book, 1997. 666p.

STORCK, L. et al. Experimentação vegetal. Santa Maria: UFSM, 2000. 198p.

THOMAS, E.J. Relationship between plot size and plot variance. Agricultural Research Journal of Kerala, v.12, p.178189, 1974.

UFV - Universidade Federal de Viçosa. SAEG - Sistema para analises estatísticas. Viçosa, 2005. Versão 9.0. CD.

VENCOVSKY, R.; BARRIGA, P. Genética biométrica no fitomelhoramento. Ribeirão Preto: Revista Brasileira de Genética, 1992. 496p.

ZANON, M.L.B. Tamanho e forma ótimos de parcelas experimentais para Eucaliptus saligna Smith. 1996. 78f. Dissertação (Mestrado em Engenharia Florestal) - Universidade Federal de Santa Maria, Santa Maria. 